\title{
Some algebraic relations between involutions, convolutions and correlations, with applications to holographic memories
}

\section{P. H. Schönemann}

Published online: 29 February 2008

(c) Springer-Verlag 2008

\section{Erratum to: Biol Cybern (1987) 56:367-374 DOI 10.1007/BF00319516}

I noticed a transposition error in the definition of $a \# b$ in Eq. (2.4) that invalidates some of the derivations on p. 369 and, in particular, the resulting summaries in Table 1. Equation (2.4) should have read

$a \# b=a^{*} S b$

and, consequently, the matrix representation, Eq. (3.8),

$a \# b=[a] S b$.
The transpose of the resulting numerical vector in Eq. (3.9) is $(2,7,13,5)$. Equations (2.7)-(2.8) should be disregarded. The corrected Cayley tables for *, \#, and the involution $S$, are given in Table 1.

The two main results of the paper remain intact:

1. Replacement of the correlation \# by $S$ and * greatly simplifies derivations, since it restores commutativity and associativity, and

2. Deconvolution with $g$-inverses rather than correlation leads to a possibly preferable alternative memory model, given in Eq. (6.1), that avoids some of the shortcoming of the traditional convolution/correlation paradigm.

Table 1 Cayley tables for *, \#, and $S$ in $\left\langle H, \#,{ }^{*}, S\right\rangle$ (row element first factor)

\begin{tabular}{|c|c|c|c|c|c|c|c|c|c|}
\hline \multicolumn{5}{|c|}{$*_{-}>\#$} & \multicolumn{5}{|c|}{$\#-->*$} \\
\hline$*$ & $a$ & $b$ & $S a$ & $S b$ & \# & $a$ & $b$ & $S a$ & $S b$ \\
\hline$a$ & $\begin{array}{l}a \# S a \\
b \# S a\end{array}$ & $a \# S b$ & $a \# a$ & $a \# b$ & $a$ & $a * S a$ & $a^{*} S b$ & $a^{*} a$ & $a^{*} b$ \\
\hline$b$ & $b \# S a$ & $b \# S b$ & $b \# a$ & $b \# b$ & $b$ & $b^{*} S a$ & $b^{*} S b$ & $b^{*} a$ & $b^{*} b$ \\
\hline$S a$ & $a \# a$ & $b \# a$ & Sa\#a & $\begin{array}{l}S a \# b \\
S b \# a\end{array}$ & $S a$ & $S\left(a^{*} a\right)$ & $S\left(a^{*} b\right)$ & $a^{*} S a$ & $S a^{*} b$ \\
\hline$S b$ & $a \# b$ & $b \# b$ & $S b \# a$ & $\begin{array}{l}S b \# b \\
S a \# b\end{array}$ & $S b$ & $S(b * a)$ & $S(b * b)$ & $S b * a$ & $b * S b$ \\
\hline
\end{tabular}

Application to convolution memories: learning:memory trace $a^{*} b$. Recall with cue $c$ : $c \#\left(a^{*} b\right)=c^{*} S\left(a^{*} b\right)=S a^{*} S b^{*} c$. If $a=c$ is used as cue and happens to be "perfectly noiselike" ( $\left.a \# a=a^{*} S a=u\right)$, then $c \#\left(a^{*} b\right)=a^{*} S\left(a^{*} S b\right)=\left(a^{*} S a\right)^{*} S S b=u^{*} b=b$, so that, in this case, $b$ is perfectly recalled

The online version of the original article can be found under doi:10.1007/BF00319516.

P. H. Schönemann $(\varangle)$

Department of Psychological Sciences, Purdue University,

West Lafayette, IN 47906, USA

e-mail:phs@psych.purdue.edu 